\title{
Religions et mondialisation : nouvelle configuration, nouveaux acteurs
}

\section{François Mabille}

\section{(2) OpenEdition \\ 12 Journals}

Édition électronique

URL : http://journals.openedition.org/assr/1500

DOI : 10.4000/assr. 1500

ISSN : $1777-5825$

Éditeur

Éditions de l'EHESS

\section{Édition imprimée}

Date de publication : 1 avril 2003

Pagination : 27-30

ISBN : 2-222-96732-5

ISSN : 0335-5985

\section{Référence électronique}

François Mabille, «Religions et mondialisation : nouvelle configuration, nouveaux acteurs », Archives de sciences sociales des religions [En ligne], 122 | avril - juin 2003, mis en ligne le 10 novembre 2005, consulté le 01 mai 2019. URL : http://journals.openedition.org/assr/1500 ; DOI : 10.4000/assr.1500 


\title{
RELIGIONS ET MONDIALISATION : NOUVELLE CONFIGURATION, NOUVEAUX ACTEURS
}

\author{
À propos de : \\ MAINUDDIN (Rolin G.), Religion and Politics in the \\ Developing World: Explosive Interactions. Ashgate, \\ North Carolina Central University, 2002, 159 p. \\ (index). \\ ESPOSITO (John L.), WATSON (Michael) eds., \\ Religion and Global Order. Cardiff, University of \\ Wales Press, 2000, 239 p. (index) (coll. «Religion, \\ Culture and Society $»)$. \\ SHIGETONI (Shinichi), ed., The State and NGOs- \\ Perspective from Asia 1. Singapour, Institute of \\ Southeast Asian Studies, 2002, 337 p. (index).
}

Il peut sembler surprenant de rassembler ces trois ouvrages au sein d'une même note critique. Deux d'entre eux portent sur des sujets dont les titres révèlent une similitude d'intérêt orienté autour des rapports politique/religion; le troisième traite d'une autre thématique et concerne les relations entre l'État et les Organisations Non Gouvernementales (Ong) en Asie. Pourtant des questionnements identiques traversent ces trois études dont les lacunes et apports s'entrecroisent par ailleurs. De quoi est-il question principalement ? De la mondialisation et de la nouvelle place de l'État, notamment celle de l'État-Providence, perçue en terme de dérégulation ou plus simplement de reconfiguration des espaces du public et du privé, éventuellement sous l'impulsion ou sous contrôle des pouvoirs publics.

C'est donc sous la figure globale du privé qu'acteurs religieux et Ong se retrouvent et que l'on peut engager des comparaisons dans leur rapport à l'État. Et c'est plus particulièrement comme acteurs sociaux bénéficiant de structures d'opportunités - et de contraintes - nouvelles que les uns et les autres sont présentés dans ces ouvrages.

Dans cette perspective, Religion and Politics in the Developing World: Explosive Interactions est l'étude la plus classique. On y trouvera d'intéressantes synthèses ordonnées autour d'études de cas relevant de différentes latitudes: 
Nicaragua, Mexique, Malawi mais aussi Israël ou encore Algérie... Les problématiques abordées sont ici habituelles bien que les contributions tentent de préciser plus spécifiquement ce moment où les relations entre acteurs politiques et acteurs religieux tournent à l'affrontement, à la violence. Le problème est néanmoins d'avoir situé ces études dans une perspective de "retour des religions », thèse dont chacun connaît aujourd'hui les limites et surtout d'avoir confiné l'analyse au choix de cas où les acteurs religieux sont systématiquement confrontés à un dysfonctionnement du politique. L'étude qui ouvre le livre, et qui porte sur l'Église catholique au Nicaragua, est ici symptomatique d'une approche générale. De même, l'exemple algérien rappelle, à juste titre, mais sans originalité non plus, comment l'islamisme radical a bénéficié d'un contexte marqué tout à la fois par la crise économique et par l'absence de pluralisme politique au sein de l'Algérie mais aussi par la « reconversion » des «vétérans afghans » d'un lieu de combat clôt vers un nouveau momentanément plus prometteur. L'ensemble fournira néanmoins un tour du monde des lieux de conflits où sont toutefois absents les acteurs religieux non abrahamiques.

Intégré au sein de la collection « Religion, Culture and Society » de l'University of Wales (UK), Religion And Global Order, édité par J.L.E. et M.W. est un ouvrage d'une autre portée, doté d'une ambition théorique, dont témoignent au demeurant les partenariats dont il est issu avec notamment la London University, Georgtown University ou encore l'Oxford Centre for Islamic Studies. Les auteurs ne se perdent pas en définition sur la mondialisation (globalization) et sur son émergence contemporaine ou au contraire sur son inscription dans un passé déjà lointain. Leur point de départ est celui d'internationalistes : les relations internationales ont basculé dans une nouvelle ère dont la mondialisation est l'une des figures éponymes à la faveur de l'effondrement de deux piliers de l'histoire du XX $\mathrm{X}^{\mathrm{e}}$ siècle : la double disparition du communisme comme alternative à la fois à l'économie de marché mais aussi à la démocratie libérale. Dans cette perspective, la mondialisation repose sur une approche assez liée politiquement à celle de Fukuyama mais qui prend également en compte les débats actuels sur la crise de l'État-Providence et la montée en puissance, en contrepartie, des acteurs économiques. C'est d'ailleurs sur ce point que les AA. font avancer la réflexion.

Si les acteurs religieux sont aujourd'hui plus audibles, c'est bien selon eux parce que pour la première fois depuis longtemps, ils se retrouvent en phase avec les attentes de la "société civile » (on établira ici le lien avec les Ong dont nous reparlerons), alors que jusqu'à présent, leurs critiques de la modernité ne rencontraient guère d'échos. Schématiquement, on dira ici que les acteurs religieux sont en convergence avec les forces montantes de l'antimondialisation, critiques tout à la fois de la prépondérance de Davos et du FMI sur les États et plus globalement sur le politique. Ce faisant, le libéralisme est heurté de plein fouet dans ses fondements théoriques : économiquement, puisque les thèmes de la justice, de la redistribution voire des présupposés des sciences économiques sont remis en cause; mais philosophiquement également puisque «apparaissent» ou plus exactement l'on prête de nouveau attention à des acteurs religieux organisés collectivement et débattant publiquement, c'est-à-dire transgressant la compréhension libérale du religieux qui confine, on le sait, ce dernier à la sphère du privé et du fors intérieur. Sur ce dernier point, Jeff Haynes remarque avec pertinence que les libéraux ne sont pas les seuls en cause et que tant les néo-marxistes que les théoriciens réalistes des 
relations internationales ont été pris de court par cette émergence : d'où le succès du « choc des civilisations » promis par Huntington.

Simultanément, la mondialisation qui est également une révolution des techniques de l'information et de la communication, favorise le redéploiement du transnationalisme religieux qui, s'il n'est pas nouveau, trouve néanmoins aujourd'hui une logistique qui lui est favorable, tout particulièrement d'ailleurs pour les acteurs issus du «Sud». Le transnationalisme repose sur une mobilité des hommes et des idées, sur des transferts de fonds également, bref, postule un décloisonnement géographique, une déterritorialisation que la logique du travail en réseau accentue. Deux exemples fournis par les AA. emportent l'adhésion : celui de la diplomatie vaticane et celui de l'islamisme radical, même si sur ce dernier sujet, on peut regretter que les analyses ne prennent pas en compte les conséquences de l'islamisme au pouvoir, travaillées en France par Olivier Roy par exemple.

Une autre thématique suscite l'intérêt : celui des sujets pris en charge par les acteurs religieux au niveau international et qui dépassent le seul registre de la protestation. Dans le domaine de la justice sociale, de l'action en faveur du développement ou encore de la thématique écologique, les acteurs religieux agissent, travaillent dans le cadre d'advocacy networks, et se montrent capables de mobilisations dépassant leurs cercles identitaires. Une analyse sur les Sommets de la Terre onusiens serait à cet égard un utile prolongement.

Toutefois, l'ouvrage présente une lacune : celle de ne guère se pencher assez précisément sur les cadres d'action que se donnent les acteurs religieux, donc sur les modalités organisationnelles de leur mobilisation antilibérale et sur les lieux de convergence avec la "société civile». C'est sur ce point que le dernier ouvrage The States and NGOS - Perspective from Asia, est un utile renfort. Car le point de départ de cet ouvrage dirigé par S.S. est bien le même que celui du précédent : celui d'une crise de l'État-Providence dans le contexte de la mondialisation, et donc l'émergence d'une reconfiguration des espaces public et privés. Mais l'ouvrage va sans doute plus loin que le précédent dans son investigation sur les acteurs privés. Alors que Religion and Global Order demeure encore largement tributaire d'une fonction protestataire et tribunicienne des acteurs religieux, les auteurs et collaborateurs de S.S. dépassent cette approche. Les Ong, ici assimilées à des associations relevant classiquement du non gouvernemental, du non lucratif, et du secteur du volontariat orienté vers le philanthropique - le rapport à une visée internationale voire l'obtention d'un statut au sein d'une institution internationale n'est malheureusement pas pris en compte - ne constituent pas simplement des instances critiques émettant des remises en cause des politiques d'intérêt public de l'État. En Asie, mais on pourrait comparer avec l'Europe ou les États-Unis, comme le suggèrent les travaux de Jean-Louis Laville -, les associations ou Ong participent de plus en plus au travail d'élaboration de cet intérêt public, prenant en charge des secteurs relevant classiquement des attributions de l'État-Providence, comme les institutions éducatives ou hospitalières. Alors que l'ouvrage de J.L.E. et M.W. met en exergue la lutte des acteurs religieux contre le libéralisme, celui de S.S. montre au contraire que les avancées du libéralisme offre de nouvelles structures d'opportunités à la sphère associative.

Intelligemment, au lieu d'opposer en termes binaires associations et État, ou associations et forces économiques, etc., S.S. dévoile les nouveaux contextes d'action qui sont disponibles : entre la «communauté », les citoyens, le marché, 
l'État et les ressources économiques, les Ong constituent un lieu privilégié de lien social et représentent des forces économiques potentielles, dans un cadre où l'État et le marché demeurent contraignants : "When the systems of market, state, and community fail to perform their resource-distributing functions properly, there is room for NGOs to emerge as a fourth-category of agents to distribute resources on their own, or to interfere with the existing distribution systems, making up for or correction their shortcomings » (p. 11).

Il en résulte une réelle complexité de la place occupée par les Ong, selon les ressources proposées par l'État (pluralisme politique ou non par exemple), par le marché et les forces économiques. Les Ong ne font pas que du plaidoyer, mais selon l'espace disponible, modifient en substance leurs critères dénominationnels : plus ou moins non lucratives - et donc plus ou moins agents économiques, plus ou moins non gouvernementales - et donc plus ou moins étatiques ou politiques, plus ou moins bénévoles et donc plus ou moins professionnelles c'est-à-dire utilisant des ressources salariées.

C'est cette perspective qu'il serait utile de reprendre pour nuancer les approches en cours de la mobilisation des acteurs religieux. Ceux-ci sont certes confrontés aux acteurs politiques, en terme de conquête ou de rivalité de pouvoir, mais il est nécessaire d'affiner l'approche et de percevoir comment dans nos sociétés, et au sein d'ères géographiques diverses, les acteurs confessionnels retrouvent une identité sociale, notamment sous la forme associative - qui n'est pas de pure revendication mais qui s'inscrit plus globalement dans les mutations provoquées par la dérégulation et la reconfiguration de l’État-Providence.

François MABILLE

Centre d'Études Interdisciplinaires des Faits Religieux EHESS 